\title{
Preamble Sampling MAC Protocols with Persistent Receivers in Wireless Sensor Networks
}

\author{
Abdelmalik Bachir, Martin Heusse, Andrzej Duda, and Kin. K. Leung
}

\begin{abstract}
We provide an analytical framework for preamble sampling techniques for MAC protocols in wireless sensor networks, from which we derive closed-form formulas for lifetime and reliability calculations. In addition to take into account transmitter behavior that controls the form and the content of the transmitted preamble, our model also considers receiver behavior that controls the duration of preamble reception in case of successful and failed reception. Along with both transmitter and receiver behavior, our model considers a non-perfect channel and thus takes into account the impacts of transmission errors and retransmissions on lifetime and reliability of preamble sampling protocols. Numerical results show that no protocol is universally optimal; that is, each protocol has its own optimal operation point that depends on the given channel and load conditions.
\end{abstract}

\section{INTRODUCTION}

Preamble sampling [2], also referred to as LPL (Low Power Listening), is a key technique used by a large number of MAC protocols to save energy in wireless sensor networks. In preamble sampling, nodes save energy by keeping their radios off most of the time to reduce idle listening. To receive frames, nodes periodically wake up for a short time to sample the channel to check whether there is an ongoing transmission on the channel. A transmission is detected when a node finds that a preamble is being transmitted, in which case it keeps its radio on to receive the data frame that is sent just after the preamble. The preamble is used to indicate that a data frame will be transmitted and is long enough to make sure that all potential receivers wake up at least once during its transmission.

Preamble sampling techniques have been extensively investigated in the literature. Many variants have been proposed to enhance the form and contents of the transmitted preamble. Protocols, hereafter referred to as preamble-frame protocols, replace the traditional specific pattern of bits composing the preamble by a series of frames. The difference between the various preamble-frame protocols depends on whether these preamble frames are control or data frames, and on whether a gap is inserted between two consecutive preamble frames or not. Control preamble-frames are usually used to inform the receiver about the remaining length of the preamble so that it can go back to sleep to save energy and wake up again just to receive the data (e.g., MFP [1]). Data preamble-frames are used to increase reliability by duplicating the same date in the preamble (e.g., DFP [1]). When gaps are used, they are used for receiving acknowledgment frames from the receiver to stop preamble transmission (e.g., WOR [3] and CSMA-MPS [4]).

The before-mentioned contributions have omitted the receiver side as they have only focused on the transmitter side by changing the form and the content of the preamble. They have also neglected transmission errors and their effects on both energy saving and reliability. In this paper, we extend the scope of existing variants of preamble sampling protocols by considering the behavior of receivers as well. Receivers may be persistent or not. While a non-persistent receiver gives up shortly after not being able to detect a preamble frame, a persistent receiver persists in reception until it receives a frame or the channel becomes clear again. Along with this extension to the receiver side, we propose a global analytical framework in which we model the lifetime and the reliability of preamble-frame protocols over non-perfect channels. We restrict the analysis to the case of persistent receivers-the case of non-persistent receivers can be easily deducted with easier derivations. For the numerical evaluation, we consider a Rayleigh fading channel.

\section{SySTEM MOdeL}

For the sake of analysis, we distinguish between a general transmission and a single transmission. A single transmission involves only the preamble and the data, whereas a general transmission may include several single retransmission attempts. Retransmissions occur in unicast communications when the transmitter does not receive an ACK frame from the receiver. The receiver continues retransmissions until an ACK frame is received or the maximum number of transmissions $n$ is reached. The reliability $p^{R}$ is the probability that a general transmission is successful. It is equal to $1-p_{f}^{n}$, where $p_{f}$ is the probability that a single transmission fails.

The lifetime $\mathcal{L}_{\circ}$ of a particular protocol 'o' is equal to $\frac{E_{\text {initial }}}{\mathcal{P}_{\mathrm{o}}}$ where $\mathcal{P}_{\circ}(\mathrm{Joule} / \mathrm{sec})$ is the average power a node consumes and $E_{\text {initial }}$ (Joule) is its initial energy. For the sake of conciseness and simplicity, we consider only the power consumed by the radio- the power consumed by other node's components can be considered with only minor modifications to the following derivations. We have $\mathcal{P}_{\circ}=\mathcal{P}_{\circ}^{t}+\mathcal{P}_{\circ}^{r}+\mathcal{P}_{\circ}^{s}$, where $\mathcal{P}_{\circ}^{t}$ (resp. $\mathcal{P}_{0}^{r}$, and $\mathcal{P}_{0}^{s}$ ) is the average power drained in transmission (resp. reception and sampling). The average power drained during preamble sampling is $\mathcal{P}_{\circ}^{s}=\frac{\mathcal{E}_{0}^{s}}{T_{\mathrm{CI}}}$, where $\mathcal{E}_{\circ}^{s}$ is the energy drained in channel sampling and $T_{\mathrm{CI}}$ is the corresponding check interval. Similarly, the average power drained during transmission is $\mathcal{P}_{\circ}^{t}=\mathcal{E}_{\circ}^{t} \cdot F_{\text {traffic }}$, and the average power drained during reception is $\mathcal{P}_{0}^{r}=\mathcal{E}_{0}^{r} \cdot \eta \cdot F_{\text {traffic }}$, where $\eta$ is the average number of neighbors and $F_{\text {traffic }}$ the average number of messages transmitted per unit time. In the case of unicast, $\eta=1$.

The energy drained in a general transmission $\mathcal{E}^{t}$ depends on whether its single transmissions failed or succeeded and 
TABLE I

Single Transmission Failure Probability

\begin{tabular}{|c||c|c|}
\hline$p^{f}$ & Broadcast & Unicast \\
\hline LPL, MFP, CSMA-MPS & $p_{d}$ & $1-\left(1-p_{d}\right)\left(1-p_{a}\right)$ \\
\hline DFP & $\frac{p_{d}}{r_{d}}\left(\frac{1-p_{d}^{r_{d}}}{1-p_{d}}\right)$ & $1-\left[1-\frac{p_{d}}{r_{d}}\left(\frac{1-p_{d}^{r_{d}}}{1-p_{d}}\right)\right]\left(1-p_{a}\right)$ \\
\hline WOR & $\frac{p_{d}}{r_{w}}\left(\frac{1-p_{d}^{r} w}{1-p_{w}}\right)$ & $\frac{q_{w}}{r_{w}}\left(\frac{1-q_{w}^{r_{w}}}{1-q_{w}}\right)$ \\
\hline
\end{tabular}

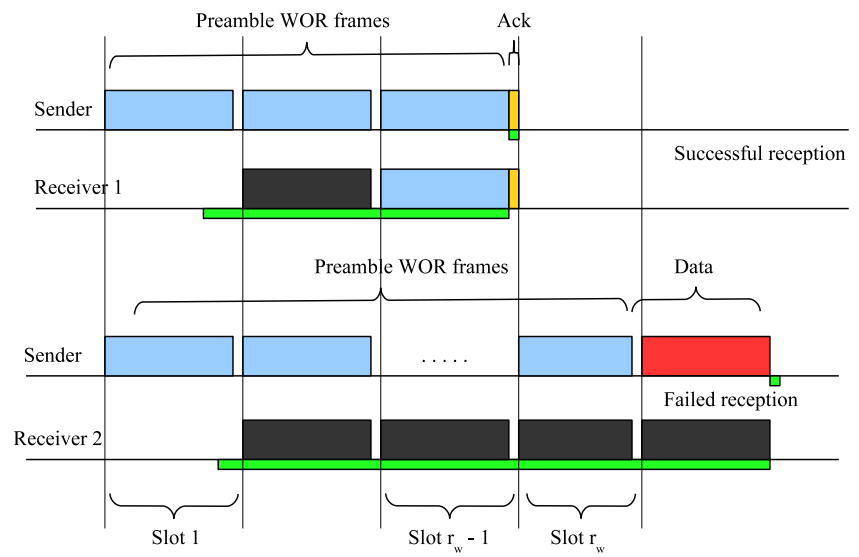

Fig. 1. Example on WOR protocol operation with a successful (Receiver 1) and failed (Receiver 2) single transmissions.

thus on the energy drained in each of these cases. We use $e_{\text {succ }}^{t}$ (resp. $e_{\text {fail }}^{t}$ ) to refer to the energy drained in the case of a successful (resp. failed) single transmission. Therefore, we have:

$$
\begin{aligned}
\mathcal{E}^{t}= & \left(1-p_{f}\right) e_{\text {succ }}^{t}+\left(1-p_{f}\right) p_{f}\left[e_{\text {fail }}^{t}+e_{\text {succ }}^{t}\right] \\
& +\cdots \\
& +\left(1-p_{f}\right) p_{f}^{n-1}\left[(n-1) e_{\text {fail }}^{t}+e_{\text {succ }}^{t}\right] \\
& +p_{f}^{n} n e_{\text {fail }}^{t} \\
= & \frac{1-p_{f}^{n}}{1-p_{f}}\left(p_{f} e_{\text {fail }}^{t}+\left(1-p_{f}\right) e_{\text {succ }}^{t}\right)
\end{aligned}
$$

The same methodology is applied to compute $\mathcal{E}^{r}$ by replacing $e_{\text {succ }}^{t}$ by $e_{\text {succ }}^{r}$ and $e_{\text {fail }}^{t}$ by $e_{\text {fail }}^{r}$ in (1), where $e_{\text {succ }}^{r}$ (resp. $e_{\text {fail }}^{r}$ ) is the average energy drained in the case of a successful (resp. failed) single reception. The energy drained in sampling $\mathcal{E}^{s}$ is independent of transmission success or failure.

\section{Evaluation of Preamble Protocols With PERSISTENT RECEIVERS}

In this section, we compute the lifetime and the reliability of preamble sampling protocols with persistent receivers. We consider five variants: LPL, MFP, DFP, WOR, and CSMAMPS for which we obtain their characteristic parameters: the probability that a single transmission fails and the energy drained in sampling, in transmission, and in reception for both unicast and broadcast communications.

\section{A. Probability of Single Transmission Failure}

The probability that a single transmission fails with LPL, MFP, and CSMA-MPS is the same. For broadcast communications, it depends only on the data frame transmitted after the preamble. In the case of unicast communications, it also depends on the ACK-frame transmitted to acknowledge reception of the data frame. For DFP and WOR, the probability of failure is different because there are copies of the data frame transmitted in the preamble and the reception of one of these frames makes the single transmission successful in the broadcast case. Therefore, a single transmission fails if the receiver cannot receive any data frame. This includes the series of preamble-frames (DFP or WOR) and the data frame transmitted afterward. As the wakeup instant of the receiver is random, it may miss the reception of $i$ DFP frames, $i=1, \ldots, r_{d}-1$, where $r_{d}$ is the number of DFP frames transmitted in the preamble to span the check interval. The value of $r_{d}$ is extracted from the check interval $T_{\mathrm{CI}}$ and the transmission duration $T_{d}$ of a DFP frame according to the following relation $r_{d}=\left\lceil T_{\mathrm{CI}} / T_{d}\right\rceil$. Therefore, if the receiver wakes up during the first DFP frame, it may keep listening during all the $r_{d}-1$ subsequent DFP frames plus the subsequent data frame if all these frames are corrupted. In this case, the number of missed frames is equal to $r_{d}$ $\left(r_{d}-1\right.$ DFP frames plus 1 data frame). As the wake up of the receiver is chosen independently of the other parameters, it can be modeled by a uniform random variable and thus the probability that the receiver wakes up during the transmission of the first, the last, or any other DFP frame is $1 / r_{d}$. Therefore, the probability $p_{f}$ of a failed single transmission is:

$$
\begin{aligned}
p_{f} & =\frac{1}{r_{d}} p_{d}^{r_{d}}+\frac{1}{r_{d}} p_{d}^{r_{d}-1}+\cdots+\frac{1}{r_{d}} p_{d} \\
& =\frac{p_{d}}{r_{d}}\left(\frac{1-p_{d}^{r_{d}}}{1-p_{d}}\right) .
\end{aligned}
$$

Note that the same formula applies for broadcast communications with WOR with replacing $r_{d}$ by $r_{w}$, which is the number of WOR frames in the preamble defined as $r_{w}=$ $\left\lceil T_{\mathrm{CI}} /\left(T_{d}+T_{a}\right)\right\rceil$, where $T_{d}$ and $T_{a}$ are the WOR and ACK transmission durations, respectively.

In the unicast case, a successful single transmission also depends on the correct reception of an ACK frame. In DFP, the ACK frame is transmitted only at the end of the transmission; therefore, the probability of failure can be derived easily from (3) as shown in Table I.

In WOR, the situation is different because an ACK frame is expected after each WOR frame transmission as shown in Fig. 1. In this case, we introduce $q_{w}$, the probability of failure within one slot (see Fig. 1) defined as:

$$
q_{w}=1-\left(1-p_{d}\right)\left(1-p_{a}\right) .
$$

Therefore, the probability of transmission failure with WOR can be derived as in (3) by replacing $p_{d}$ by $q_{w}$ and $r_{d}$ by $r_{w}$. 


$$
\begin{array}{rlrl}
P\left[Y_{w}=2\right] & =\frac{1}{r_{w}}\left(1-q_{w}\right)+\frac{1}{r_{w}} 0+\cdots+\frac{1}{r_{w}} 0 & & \frac{1}{r_{w}}\left(1-q_{w}\right) \\
P\left[Y_{w}=3\right] & =\frac{1}{r_{w}} q_{w}\left(1-q_{w}\right)+\frac{1}{r_{w}}\left(1-q_{w}\right)+\frac{1}{r_{w}} 0+\cdots+\frac{1}{r_{w}} 0 & =\frac{1}{r_{w}}\left(1-q_{w}^{2}\right) \\
\vdots & \vdots & \underbrace{}_{r_{w}-(i-1) \text { times }} & \\
P\left[Y_{w}=i\right] & =\frac{1}{r_{w}} q_{w}^{i-2}\left(1-q_{w}\right)+\cdots+\frac{1}{r_{w}} q_{w}\left(1-q_{w}\right)+\frac{1}{r_{w}} 0+\cdots+\frac{1}{r_{w}} & = & \frac{1}{r_{w}}\left(1-q_{w}^{i-1}\right) \\
\vdots= & \vdots \\
P\left[Y_{w}=r_{w}-1\right] & =\frac{1}{r_{w}} q_{w}^{r_{w}-3}\left(1-q_{w}\right)+\cdots+\frac{1}{r_{w}}\left(1-q_{w}\right)+\frac{1}{r_{w}} 0 & & \frac{1}{r_{w}}\left(1-q_{w}^{r_{w}-2}\right) \\
P\left[Y_{w}=r_{w}\right] & =\frac{1}{r_{w}} q_{w}^{r_{w}-2}+\cdots+\frac{1}{r_{w}} q_{w}+\frac{1}{r_{w}}+\frac{1}{r_{w}} & & \frac{1}{r_{w}}\left(1+\frac{1-q_{w}^{r_{w}-1}}{1-q_{w}}\right)
\end{array}
$$

TABLE II

SAMPLING COST

\begin{tabular}{|c||c|}
\hline $\mathcal{E}^{s}$ & Broadcast or Unicast \\
\hline LPL, MFP, DFP & $\left(\tau+T_{\mathrm{CS}}\right) P_{s}$ \\
\hline CSMA-MPS, WOR & $\left(\tau+T_{a}+T_{\mathrm{CS}}\right) P_{s}$ \\
\hline
\end{tabular}

Table I summarizes these probabilities. Note that $p_{a}$ refers to the probability that an ACK frame is corrupted.

\section{B. Energy Drained in Channel Sampling}

The energy drained in sampling operation depends on $\tau$, the time needed to switch the radio from sleep to receive state ${ }^{1}$, and on the time needed to draw a conclusion on whether the channel is active or not. The latter time includes $T_{\mathrm{CS}}$, the time needed to perform a RSSI measure and includes the inter preamble frame time $T_{a}$ envisaged for ACK frames if gaps are inserted between them such as in CSMA-MPS and WOR. The time needed for channel sampling is independent of whether the transmission is broadcast or unicast. Table II summarizes the obtained results for the considered preamble sampling variants. We have used $P_{s}$ to refer to the power drained when the radio is in sampling mode.

\section{Energy Drained in Transmission}

As in most of contention-based protocols, each transmission in preamble sampling protocols is preceded by a carrier sense operation. Carrier sensing before transmission is exactly similar to the channel sampling operation and thus the drained energy is equal to the corresponding $\mathcal{E}^{s}$. For protocols without inter preamble-frame gaps such as LPL, MFP, and DFP, the energy drained by the transmitter is the same independently of whether the single transmission fails or succeeds. It includes the transmission of all preamble frames and the subsequent data frame. For broadcast communications with WOR and CSMA-MPS, the preamble is transmitted entirely, because no

${ }^{1}$ In contrast to other transitions, this transition cannot be neglected. For the CC 2500 radio, the transition from sleep mode to active mode is $88.4 \mu \mathrm{s}$, whereas the transition from receive to transmit mode is only $9.6 \mu \mathrm{s}$.
ACK are expected. We have thus assumed that the transmitter goes back to sleep mode during the inter preamble-frame gaps. For unicast communications, we introduce a random variable $Y$ that counts the number of preamble frames transmitted. The transmission of preamble frames stops when the transmitter receives an ACK frame from the receiver that acknowledges the correct reception of the just transmitted preamble frame. Note that the minimum number of transmitted preamble frames is 2 because in the best case, the receiver wakes up in slot 1 and thus misses the first preamble frame. In the case of WOR, the random variable $Y$ is called $Y_{w}$ and is defined in $\left\{2, \ldots, r_{w}\right\}$ as in (2). Note that the $P\left[Y_{w}=r_{w}\right]$ has a different form from the others. Thus, we have:

$$
P\left[Y_{w}=i\right]= \begin{cases}\frac{1}{r_{w}}\left(1-q_{w}^{i-1}\right) & \text { if } 2 \leq i<r_{w} \\ \frac{1}{r_{w}}\left(1+\frac{1-q_{w}^{r_{w}-1}}{1-q_{w}}\right) & \text { if } i=r_{w} .\end{cases}
$$

The value of $Y_{x}$ for CSMA-MPS is obtained in a similar way. Table III summarizes the obtained results. Variables $P_{t}$ and $P_{r}$ refer to the power the radio consumes in transmit and receive modes, respectively.

\section{Energy Drained in Reception}

The reception starts when a node detects that a preamble is being transmitted. As the receiver may wake up at any time during preamble transmission, it can only receive the remaining part of the preamble. In the case of LPL, it receives half of the preamble on the average. In the case of preambleframe protocols, it receives a number of preamble frames. In general, the receiver is not guaranteed to wake up right at the beginning of each preamble-frame, thus the first detected preamble-frame is missed. On the average, only half of it is received. For derivations, we consider both cases of successful and failed single reception: $e_{\text {succ }}^{r}$ and $e_{\text {fail }}^{r}$, respectively. Table IV summarizes the obtained results. Note that the energy drained in reception also includes the energy drained when the radio switches from sleep to receive mode.

To compute the energy drained in preamble reception, we introduce a protocol-specific random variable $X$ that counts 
TABLE III

Single TRANSMisSion Cost

\begin{tabular}{|c|c|c|}
\hline$e^{t}$ & Broadcast & Unicast \\
\hline LPL & $e_{\mathrm{succ}}^{t}=e_{\mathrm{fail}}^{t}=\mathcal{E}^{s}+\left(T_{\mathrm{CI}}+T_{d}\right) P_{t}$ & $e_{\mathrm{succ}}^{t}=e_{\mathrm{fail}}^{t}=\mathcal{E}^{s}+\left(T_{\mathrm{CI}}+T_{d}\right) P_{t}+T_{a} P_{r}$ \\
\hline MFP & $e_{\text {succ }}^{t}=e_{\text {fail }}^{t}=\mathcal{E}^{s}+\left(r_{m} T_{m}+T_{d}\right) P_{t}$ & $e_{\text {succ }}^{t}=e_{\text {fail }}^{t}=\mathcal{E}^{s}+\left(r_{m} T_{m}+T_{d}\right) P_{t}+T_{a} P_{r}$ \\
\hline DFP & $e_{\mathrm{succ}}^{t}=e_{\text {fail }}^{t}=\mathcal{E}^{s}+\left(r_{d} T_{d}+T_{d}\right) P_{t}$ & $e_{\mathrm{succ}}^{t}=e_{\mathrm{fail}}^{t}=\mathcal{E}^{s}+\left(r_{d} T_{d}+T_{d}\right) P_{t}+T_{a} P_{r}$ \\
\hline WOR & $e_{\text {succ }}^{t}=e_{\text {fail }}^{t}=\mathcal{E}^{s}+\left[r_{w}\left(\tau+T_{d}\right)+T_{d}\right] P_{t}$ & $\begin{aligned} e_{\text {succ }}^{t} & =\mathcal{E}^{s}+\overline{Y_{w}}\left(T_{d} P_{t}+T_{a} P_{r}\right)+T_{d} P_{t}+T_{a} P_{r} \\
e_{\text {fail }}^{t} & =\mathcal{E}^{s}+r_{w}\left(T_{d} P_{t}+T_{a} P_{r}\right)+T_{d} P_{t}+T_{a} P_{r}\end{aligned}$ \\
\hline CSMA-MPS & $e_{\text {succ }}^{t}=e_{\text {fail }}^{t}=\mathcal{E}^{s}+\left[r_{x}\left(\tau+T_{x}\right)+T_{d}\right] P_{t}$ & $\begin{aligned} e_{\mathrm{succ}}^{t} & =\mathcal{E}^{s}+\overline{Y_{x}}\left(T_{x} P_{t}+T_{a} P_{r}\right)+T_{d} P_{t}+P_{a} T_{r} \\
e_{\text {fail }}^{t} & =\mathcal{E}^{s}+r_{x}\left(T_{x} P_{t}+T_{a} P_{r}\right)+T_{d} P_{t}+P_{a} T_{r}\end{aligned}$ \\
\hline
\end{tabular}

TABLE IV

SINGLE RECEPTION COST

\begin{tabular}{|c|c|c|}
\hline$e^{r}$ & Broadcast & Unicast \\
\hline LPL & $e_{\mathrm{succ}}^{r}=e_{\mathrm{fail}}^{r}=\left(\tau+T_{\mathrm{CI}} / 2+T_{d}\right) P_{r}$ & $\begin{array}{l}e_{\mathrm{succ}}^{r}=\left(\tau+T_{\mathrm{CI}} / 2+T_{d}\right) P_{r}+T_{a} P_{t} \\
e_{\mathrm{fail}}^{r}=\left(\tau+T_{\mathrm{CI}} / 2+T_{d}\right) P_{r}+\left(1-p_{d}\right) T_{a} P_{t}\end{array}$ \\
\hline MFP & $\begin{array}{l}e_{\text {succ }}^{r}=\left(\tau+T_{m} / 2+\overline{X_{m}} T_{m}+\tau+T_{d}\right) P_{r} \\
e_{\text {fail }}^{r}=\left(\tau+T_{m} / 2+\overline{X_{m}} T_{m}+T_{d}\right) P_{r}\end{array}$ & $\begin{array}{l}e_{\text {succ }}^{r}=\left(\tau+T_{m} / 2+\overline{X_{m}} T_{m}+\tau+T_{d}\right) P_{r}+T_{a} P_{t} \\
e_{\text {fail }}^{r}=\left(\tau+T_{m} / 2+\overline{X_{m}} T_{m}+T_{d}\right) P_{r}+\left(1-p_{d}\right) T_{a} P_{t}\end{array}$ \\
\hline DFP & $e_{\text {fail }}^{r}=e_{\text {succ }}^{r}=\left(\tau+T_{d} / 2+\overline{X_{d}} T_{d}+T_{d}\right) P_{r}$ & $\begin{array}{l}e_{\text {succ }}^{r}=\left(\tau+T_{d} / 2+\overline{X_{d}} T_{d}+T_{d}\right) P_{r}+T_{a} P_{t} \\
e_{\text {fail }}^{r}=\left(\tau+T_{d} / 2+\overline{X_{d}} T_{d}+T_{d}\right) P_{r}+ \\
\quad\left[1-\frac{p_{d}}{r_{d}}\left(\frac{1-p_{d}^{r_{d}}}{1-p_{d}}\right)\right]\left(\tau+T_{a}\right) P_{t}\end{array}$ \\
\hline WOR & $\begin{array}{l}e_{\text {fail }}^{r}=e_{\text {succ }}^{r}= \\
{\left[\tau+\left(T_{a}+T_{d}\right) / 2+\left(T_{a}+T_{d}\right) \overline{X_{w}}+T_{d}\right] P_{r}}\end{array}$ & $\begin{aligned} e_{\mathrm{succ}}^{r}= & {\left[\tau+\left(T_{a}+T_{d}\right) / 2\right] P_{r}+\overline{X_{w}}\left[p_{d}\left(T_{d}+\tau\right) P_{r}+\right.} \\
& \left.\left(1-p_{d}\right)\left(T_{d} P_{r}+T_{a} P_{t}\right)\right]+T_{d} P_{r}+T_{a} P_{t} \\
e_{\text {fail }}^{r}= & {\left[\tau+\left(T_{a}+T_{d}\right) / 2\right] P_{r}+\overline{X_{w}}\left[p_{d}\left(T_{d}+\tau\right) P_{r}+\right.} \\
& \left.\left(1-p_{d}\right)\left(T_{d} P_{r}+T_{a} P_{t}\right)\right]+T_{d} P_{r}+\left(1-p_{d}\right) T_{a} P_{t}\end{aligned}$ \\
\hline CSMA-MPS & $\begin{array}{r}e_{\text {fail }}^{r}=e_{\text {succ }}^{r}=\left[\tau+\left(T_{a}+T_{x}\right) / 2+\right. \\
\left.\left(T_{a}+T_{x}\right) \overline{X_{x}}+\left(\tau+T_{d}\right)\right] P_{r}\end{array}$ & $\begin{aligned} e_{\mathrm{succ}}^{r}= & {\left[\tau+\left(T_{a}+T_{x}\right) / 2\right] P_{r}+\overline{X_{x}}\left[T_{x} P_{r}\right.} \\
& \left.\left(1-p_{x}\right) T_{a} P_{t}+p_{x} \tau P_{r}\right]+\left(\tau+T_{d}\right) P_{r}+T_{a} P_{t}+ \\
e_{\mathrm{fail}}^{r}= & {\left[\tau+\left(T_{a}+T_{x}\right) / 2\right] P_{r}+\overline{X_{x}}\left[T_{x} P_{r}+\right.} \\
& \left.\left(1-p_{x}\right) T_{a} P_{t}+p_{x} \tau P_{r}\right]+T_{d} P_{r}+\left(1-p_{d}\right) T_{a} P_{t}\end{aligned}$ \\
\hline
\end{tabular}

the number of received preamble-frames. For protocols that use control frames such as MFP and CSMA-MPS, $X$ counts the number of all received preamble-frames, which is a succession of corrupted preamble frames followed by a successful preamble frame. For MFP, the variable is called $X_{m}$ and is defined in $\left\{0, r_{m}-1\right\}$. We have $P\left[X_{m}=0\right]=1 / r_{m}$, because the probability of receiving 0 MFP frame is the probability that the receiver wakes up during the last MFP frame transmission. This wake up is independent and thus can be assumed uniform in the general case. Hence, the probability is equal to $1 / r_{m}$. To compute $P\left[X_{m}=i\right]$ for $i \in\left\{1, r_{m}-1\right\}$, we use the relation $P\left[X_{m}=i\right]=P\left[X_{m} \geq i\right]-P\left[X_{m} \geq i+1\right]$. We have:

$$
\begin{aligned}
P\left[X_{m} \geq i\right]= & \underbrace{\frac{1}{r_{m}} p_{m}^{i}+\cdots+\frac{1}{r_{m}} p_{m}^{i}}_{\text {If the receiver wakes up before position } r_{m}-i} \\
& +\underbrace{\frac{1}{r_{m}} 0+\cdots \frac{1}{r_{m}} 0}_{\text {Otherwise }} \\
= & \frac{r_{m}-i}{r_{m}} p_{m}^{i}
\end{aligned}
$$

Therefore,

$$
P\left[X_{m}=i\right]=\frac{r_{m}-i}{r_{m}} p_{m}^{i-1}-\frac{r_{m}-(i+1)}{r_{m}} p_{m}^{i} .
$$

The derivation of $X_{x}$ is similar to that of $X_{m}$ with replacing $r_{m}$ by $r_{x}$ and $p_{m}$ by $p_{x}$ (where $p_{x}$ is the probability that a CSMA-MPS preamble frame is corrupted).

For protocols that use data frames in the preamble, $X$ counts the number of corrupted data frames received in the preamble. The derivations are similar to those described above. For DFP, we have:

$$
P\left[X_{d}=i\right]=\frac{r_{d}-i}{r_{d}} p_{d}^{i}-\frac{r_{d}-(i+1)}{r_{d}} p_{d}^{i+1} .
$$

Similarly, $X_{w}$ is obtained by replacing $r_{d}$ by $r_{w}$.

For the unicast case, the ACK frames should be taken into account. That is, $p_{d}$ is replaced by $q_{w}$ in the calculation of $X_{w}$, and $p_{x}$ by $q_{x}$ in the calculation of $X_{x}$. Detailed results are summarized in Table IV.

\section{NumericAl RESUlts}

For the numerical evaluation, we consider a Rayleigh fading channel and use a familiar model to compute the packet error probability [5]. For other parameters, we use the characteristics 


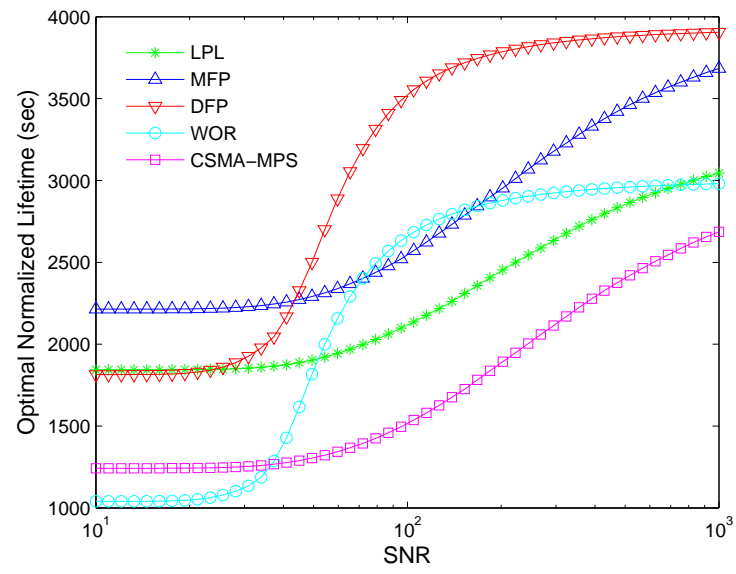

(a) Optimal Normalized Lifetime.

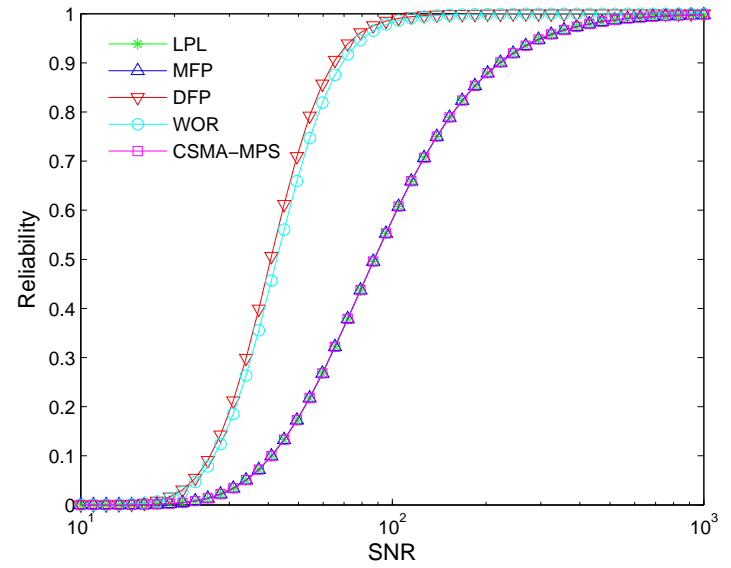

(b) Communication Reliability.

Fig. 2. Optimal Normalized Lifetime and Reliability for Unicast Communications

of the CC 2500 radio [3]. We set control frames to 16 bytes and data frames to 128 bytes. We consider a unicast communication link with a maximum of 2 retransmissions in case of failure (i.e., $n=3$ ). We evaluate two main parameters: reliability and lifetime as shown in Fig. 2(a) and Fig. 2(b), respectively. For reliability, we clearly show that protocols with duplicated data frames in the preamble (WOR and DFP) are more reliable than the others. For lifetime, we plot the maximum lifetime, obtained when an optimal check interval $\left(T_{\mathrm{CI}}\right)$ is used, for each protocol. Fig. 2(a) shows that protocols with inter preamble-frame gaps (i.e., WOR and CSMA-MPS) are not always the best candidates as it could have been concluded from the analysis of only transmission and reception costs presented in Section III. Fig. 2(a) shows that protocols without inter preamble-frame gaps (LPL, MFP, and DFP) have longer lifetimes than the others (WOR and CSMA-MPS). This result points out the importance of the energy drained in channel sampling. In WOR and CSMA-MPS, the time needed for channel sampling is increased because of the inter preambleframe gaps. Therefore, the energy those protocols save by cutting the full-length preamble in transmission is dominated by the energy wasted in longer channel-sampling times. Even for relatively high traffic loads for sensor networks, such as 1 message per minute used for plotting Fig. 2(a), the sampling cost is dominant.

\section{Conclusions}

We have taken into account non-perfect channel conditions to compute the lifetime and the reliability of various preamble sampling techniques with persistent receivers. Our numerical results show that the channel sampling cost has a significant impact on the energy consumption of sampling protocols. Moreover, no protocol maximizes reliability and lifetime at the same time. Therefore, no protocol is universally optimaleach one has its own optimal operation point that depends on given channel and load conditions.

\section{ACKNOWLEDGMENT}

This work is funded in part by the French Ministry of Research project ARESA under contract ANR-05-RNRT-01703 and UK EPSRC Research Grant EP/D076838/1 entitled Smart Infrastructure: Wireless Sensor Network System for Condition Assessment and Monitoring of Infrastructure. Special thanks to Mischa Dohler and reviewers for their comments and useful suggestions.

\section{REFERENCES}

[1] A. Bachir, L. Samper, D. Barthel, M. Heusse and A. Duda. Link Cost and Reliability of Frame Preamble MAC Protocols. In Proceedings of IWWAN, New York, NY, June 2006.

[2] A. El-Hoiydi. Aloha with Preamble Sampling for Sporadic Traffic in Ad Hoc Wireless Sensor Networks. In Proceedings of IEEE ICC, New York, NY, April 2002.

[3] Chipcon Corporation. CC2500 Single Chip Low Cost Low Power RF Transceiver, Data Sheet. 2005.

[4] S. Mahlknecht and M. Boeck. CSMA-MPS: A Minimum Preamble Sampling MAC Protocol for Low Power Wireless Sensor Networks. In Proceedings of IEEE Workshop on Factory Communication Systems, Vienna, Austria, September 2004.

[5] D. Tse and P. Viswanath. Fundamentals of Wireless Communication. Cambridge university press edition, 2005. 